\title{
Neurologists may Perform Interventional Neuroangiography
} Nörologlar Girişimsel Nöroanjiyografi Yapabilmelidir

\section{Neurologists may Perform Interventional Neuroangiography}

Definitely "Yes". The base of this clear answer is very reliable. The year 2015 witnessed an important improvement in medicine. Owing to the results of 7 randomized trials published in 2015, endovascular recanalization (thrombectomy/thromboaspiration) became the standard treatment of acute ischemic stroke due to large vessel occlusion with level of class IA evidence. As a result of this improvement, the consensus document of 12 international multi-disciplinary neurointerventional societies, which contains training guidelines who would treat patients with acute ischemic stroke, was published in 8 international journals simultaneously (1). A need for well-trained neurointerventionalists to perform acute ischemic stroke treatment in an organized and efficient team, is cited in the article. A neuroscience background and dedicated neurointerventional training are critical to ensure the best possible patient outcomes.

Recently, a meta-analysis showed that most thrombectomy procedures are performed by experienced neurointerventionalists, including interventional neuroradiologists, endovascular neurosurgeons or interventional neurologists in patients with acute ischemic stroke (2). Existence of vascular neurology specialists and neuro-critical care units are reported to be associated with better patient outcomes.

The answer "Yes" can be supported with another point of view. An analogy can be made between an interventional neurologist and an interventional cardiologist. A cardiologist can perform echocardiography, give medical treatment, monitor patients, and perform angiography if necessary. It would be reasonable if a neurologist did similar treatments. Acute ischemic stroke is a complex disorder. A successful endovascular treatment is based on quick and comprehensive integration of patient's anamnesis, clinical and neuroradiological findings. For a good outcome, appropriate patient selection and interventional specialization are required. As a consequence, formal training for both clinical neuroscience and interventional neuroradiology are required. According to the consensus document mentioned above, neuroradiologists, neurologists, and neurosurgeons can be specialized in interventional neurology for endovascular treatment of acute ischemic stroke. The duration of training is two years. The first year of training includes clinical neuroscience and neuroimaging. The second year includes interventional neuroradiology based on endovascular treatment of ischemic stroke under the supervision of a competent interventional neuroradiologist, interventional neurologist or endovascular neurosurgeon in a high volume center. Those who were trained for clinical neuroscience, diagnostic neuroradiology, and neuroangiogiaphy during their specialization do not need to take the first year of the training. These remarkable recent improvements indicate that neurologists who were trained well, can perform interventional neuroangiography.

As an interventional neurologist who began to study catheterbased cerebral angiography 45 years ago and became the pioneer of interventional neuroangiography in Turkey in the 1980s after getting trained abroad, I'm sure you'll anticipate the level of happiness I have, when I see these improvements. But I have a concern that I want to mention. In this "interventional neuroangiography" field, neurologists have to compete against interventional neuroradiologists and endovascular neurosurgeons by means of knowledge, experience and capability and I fear that neurologists may fall behind. But this competition should not be for grabbing a place in this field. They should work as a team. I think, a vascular neurologist (also can be an interventional neurologist) and an interventionalist (neuroradiologist, 
neurosurgeon or neurologist) should get involved in a process of endovascular treatment of a patient with acute ischemic stroke. An interventional neurologist is mainly a vascular neurologist and has the advantage of having a better clinical neuroscience background. In contrast, interventional neurologists have less experience and capability in manual manipulation compared with the other two disciplines, which is a disadvantage. Therefore, depending on the center in which they are trained, the duration of interventional neuroangiography training could last longer than 12 months. With the requests of some young neurologists who are interested in neuroangiography, the "Neuroradiology Scientific Working Group” was founded by the Turkish Society of Neurology (TND) in January 2009 and was selected as a moderator. The name was then changed to the "Interventional Neurology Scientific Working Group”. The TND Cerebrovascular Diseases Scientific Working Group, the TND Neuro-Critical Care Scientific Working Group, and the Turkish Cerebrovascular Diseases Society offered a "Vascular Neurology Sub-branch Speciality", which lasts 3 years and includes interventional neuroangiography. This offer was delivered to the Ministry of Health by the two societies. These societies exported "Vascular Neurology Physician Certificates" to the experienced neurologists who could give the vascular neurology training. Considering the fact that the proposal given to the Ministry of Medicine will not result in the near term, the TND Interventional Neurology Scientific Working Group specified the training criteria for "Diagnostic Neuroangiography" and "Interventional Neuroangiography" and released them on the TND's web site. These criteria were revised in consideration of the consensus document of international multi-disciplinary neurointerventional societies. After completing the training for ischemic stroke treatment, interventional neurologists can also perform cervico-cerebral stent/angioplasty (especially carotid), in addition to thrombectomy/thromboaspiration. After taking extra training, an interventional neurologist can even treat aneursyms, arterio-venous malformations, and perform tumor embolizations. The path of training for "Interventional Neurology" was opened clearly in the world in 2016. In Turkey, the seeds of "Interventional Neurology" were sown in 2009.

I want to end this article by highlighting the fact that neuroendovascular interventions, which can have a high risk of morbidity and complication, should be performed by experienced physicians who were trained by competent specialists in appropriate centers.

\section{Etbics}

Peer-review: Internal peer-reviewed.

\section{References}

1. Training guidelines for endovascular ischemic stroke intervention: An international multi-society consensus document EJMINT editorial, 2016: 1607000288 (18th February 2016). http://ine.sagepub.com/content/ early/2016/03/07/1591019916636801.full.pdf

2. Badhiwala JH, Nassiri F, Alhazzani W, Selim MH, Farrokhyar F, Spears J, Kulkarni AV, Singh S, Alqahtani A, Rochwerg B, Alshahrani M, Murty NK, Alhazzani A, Yarascavitch B, Reddy K, Zaidat OO, Almenawer SA. Endovascular thrombectomy for acute ischemic stroke: A meta-analysis. JAMA 2015;314:1832-1843. 